\title{
Editorial
}

\section{Ecological monitoring, assessment, and restoration of running waters in Korea}

\author{
Soon-Jin Hwang ${ }^{1 *}$, Sang-Woo Lee ${ }^{1}$ and Young-Seuk Park ${ }^{2}$ \\ ${ }^{1}$ Department of Environmental Science, Konkuk University, Seoul 143-701, Republic of Korea \\ 2 Department of Biology, Kyung Hee University, Seoul 130-701, Republic of Korea
}

Received 10 March 2011; Accepted 19 March 2011

In most parts of the world, anthropogenic disturbances have significantly impacted various ecosystems, beginning in the previous century. Urbanization, industrialization, and land transformation have dramatically altered many such systems, and have resulted in the development of many kinds of "emerging ecosystems" (e.g., Milton, 2003), often leaving indelible ecological footprints on the Earth's surface. Dramatic alteration of aquatic habitats and biological degradation of streams and rivers have been observed worldwide. The pervasive nature of these phenomena demonstrates that traditional technology-based approaches, in particular efforts to treat wastewater, have failed to protect the quality of water resources. Until recently, many countries have managed water resources using mainly chemical criteria (e.g., biochemical oxygen demand, chemical oxygen demand, total phosphorus and nitrogen levels) of water quality, with scant regard being paid to the welfare of aquatic organisms and the biological integrity of ecosystems.

Korea only recently adopted biological criteria of water quality and the concept of ecological integrity. Such concerns are now part of the policy of surface water management, and a Nationwide Aquatic Ecological Monitoring Program (NAEMP) has been established. In the course of program development, the Korean government developed monitoring guidelines and assessment tools, including various biological and habitat indices. This work was done between 2003 and 2006, and nationwide monitoring has been conducted every year since 2007. Such monitoring includes evaluation of the chemical, physical, and biological characteristics of river/stream ecosystems. One of the most striking features of the NAEMP is that three trophic levels (benthic diatoms, benthic macroinvertebrates, and fish) of a stream food chain are monitored at the same sampling sites at the same time. Conventional environmental factors are also measured. Many research scientists and experts in the fields of biology, limnology, landscape ecology, restoration ecology, and geographic

\footnotetext{
*Corresponding author: sjhwang@konuk.ac. kr
}

information system and data management participate in the program.

Despite rapid technological advances in data collection and analysis, which have widened our ability to explore complex ecosystems and their dynamic responses to anthropogenic disturbances, knowledge of how ecosystems respond to such perturbations remains limited. As the complexity of a particular ecosystem can be more clearly understood as knowledge accumulates, systematic longterm monitoring is crucial. Thus, the Korean experience with NAEMP is unique, and provides a useful framework for assessing and managing water resources. This program also offers invaluable opportunities for exploration, at many levels, of the dynamic and complex relationships between anthropogenic disturbances and their ecological impacts on streams and rivers.

The results described in this special issue form part of the "Survey and Evaluation of Aquatic Ecosystem Health in Korea" and focus principally on data gathered in 2009. The special issue contains nine articles. First, the monitoring program is introduced, and the biological characteristics, ecological assessment, and restoration of Korean rivers and streams are described. The most important finding to date is that many Korean rivers and streams are severely degraded, such that they are in a biological condition much worse than conventional water chemistry data would suggest.

To briefly summarize the major points made by the articles in this special issue, S.-W. Lee et al. (2011) offer a short history of the NAEMP in Korea; cover the development of the required indicators, metrics, and sampling protocol; describe some key findings; and consider how monitoring results have been applied. Hwang et al. (2011) and Yoon et al. (2011) describe the nationwide distributions of benthic diatom flora and fish fauna, respectively, with regard to environmental gradients. Two articles address land use effects on various characteristics of running water ecosystems. Jun et al. (2011) explore how land use practices affect macroinvertebrate communities. Park et al. (2011) consider the relationship between land 
use in watersheds and variations in monitored biological indices at two different spatial scales, and draw the conclusion that the overall biological integrity of rivers/ streams is more sensitive to land use at the regional rather than the site scale. Ecological health assessment and restoration are important topics in this special issue. Cho et al. (2011) consider the various metrics used to monitor benthic macroinvertebrates, in terms of stream biological water quality and ecosystem health, and test their utility in comparison with conventional indicators. J.H. Lee et al. (2011) assess river ecological health using indices derived from fish and the habitat. Bae et al. (2011) emphasize that the concept of multiscale habitat filters is very useful when stream functional organization is to be evaluated, and strongly recommend that multiple biological indices be employed when assessing the biotic integrity of aquatic ecosystems. Jeong et al. (2011) explore the general patterns of anthropogenic disturbance in Korean streams using a stream modification index.

Acknowledgements. We are very grateful to all the contributors to this special issue, to the members of the monitoring project, and to the reviewers for their invaluable comments that improved the scientific quality of all manuscripts. Our special thanks go to the Ministry of Environment and the National Institute of Environmental Research for providing the financial support that has made this special issue possible.

\section{References}

Bae M.J., Kwon Y.S., Hwang S.-J., Chon T.-S., Yang H.J., Kwak I.-S., Park J.-H., Ham S.-A. and Park Y.-S., 2011. Relationships between three major stream assemblages and their environmental factors in multiple spatial scales. Ann. Limnol. - Int. J. Limnol., 47, S91-S105.

Cho W.S., Park Y.-S., Park H.-K., Kong H.-Y. and Chon T.-S., 2011. Ecological informatics approach to screening of integrity metrics based on benthic macroinvertebrates in streams. Ann. Limnol. - Int. J. Limnol., 47, S51-S62.

Hwang S.-J., Kim N.Y., Yoon S.A., Kim B.-H., Park M.H., You K.-A., Lee H.Y., Kim H.S., Kim Y.J., Lee J.H., Lee O.M., Shin J.-K., Lee E.J., Jeon S.L. and Joo H.S., 2011. Distribution of benthic diatoms in Korean rivers and streams in relation to environmental variables. Ann. Limnol. - Int. J. Limnol., 47, S15-S33.

Jeong K.S., Kim H.-G., Jeong J.-C., Kim D.-K., Kim H.-W., Lee J.-K. and Joo G.-J., 2011. Current status of Korean stream and exploring areas with high necessity for stream structure restoration. Ann. Limnol. - Int. J. Limnol., 47, S117-S125.

Jun Y.-C., Kim N.Y., Kwon S.-J., Han S.-C., Hwang I.-C., Park J.-H., Won D.-H., Byeon M.-S., Kong H.-Y., Lee J.E. and Hwang S.-J., 2011. Effects of land use on benthic macroinvertebrate communities: comparison of two mountain streams in Korea. Ann. Limnol. - Int. J. Limnol., 47, S35-S49.

Lee J.H., Han J.-H., Kumar H.K., Choi J.-K., Byeon H.K., Choi J.S., Kim J.-K., Jang M.-H., Park H.-K. and An K.-G., 2011. National-level integrative ecological health assessments based on the index of biological integrity, water quality, and qualitative habitat evaluation index, in Korean rivers. Ann. Limnol. - Int. J. Limnol., 47, S73-S89.

Lee S.-W., Hwang S.-J., Lee J.-K., Jung D.-I., Park Y.-J. and Kim J.T., 2011. Overview and application of the National Aquatic Ecological Monitoring Program (NAEMP) in Korea. Ann. Limnol. - Int. J. Limnol., 47, S3-S14.

Milton S.J., 2003. "Emerging ecosystems": a washing-stone for ecologists, economists and sociologists? S. Afr. J. Sci., 99, 404-406.

Park S.-R., Lee H.-J., Lee S.-W., Hwang S.-J., Byeon M.-S., Joo G.-J., Jeong K.-S., Kong D.-S. and Kim M.-C., 2011. Relationships between land use and multi-dimensional characteristics of streams and rivers at two different scales. Ann. Limnol. - Int. J. Limnol., 47, S107-S116.

Yoon J.-D., Kim J.-H., Byeon M.-S., Yang H.-J., Park J.-Y., Shim J.-H., Song H.-B., Yang H. and Jang M.-H., 2011. Distribution patterns of fish communities with respect to environmental gradients in Korean streams. Ann. Limnol. Int. J. Limnol., 47, S63-S71. 